\title{
Grey Relative Evaluation of Airport Apron Safety Risk based on THEIL Index
}

\author{
Shi $\operatorname{Li}^{1,2,3}$ and Luo Fan ${ }^{1, *}$ \\ ${ }^{1}$ School of management, Wuhan University of Technology, Wuhan, 430070, \\ China \\ ${ }^{2}$ School of Information Engineering, Hubei University of Economics, Wuhan, \\ 430205, China \\ ${ }^{3}$ Department of Management Science and Information Systems, Rutgers \\ University, New Jersey, America \\ Corresponding Author: Fan Luo, School of management, Wuhan University of \\ Technology, Wuhan, 430070, China
}

\begin{abstract}
Aiming at the airport apron safety risk evaluation, using a comprehensive integrated view, combining the quantitative method with qualitative method, a grey relative comprehensive evaluation model of apron safety risk based on THEIL index is proposed. First, objective weight is calculated by THEIL index, combined with experience and knowledge of the subjective weight to determine the final comprehensive weight. Then, based on comprehensive weight, the fuzzy evaluation and grey correlation are used to obtain the results of degree of association, and specific risk evaluation order of aprons is achieved. Finally, the validity of the model is verified by an example, and it provides an effective method for the evaluation of airport apron safety risk.
\end{abstract}

Keywords: Airport apron safety, THEIL index, Grey relational evaluation

\section{Introduction}

Airport security management is an important part of aviation safety management. It directly affects the safety of aircraft operations on the ground and the efficiency of onland guarantee operations. With the high-speed development of the air transport volume, there are many obvious characteristics about apron operation such as many units, traffic tension, work busy and so on, thus demand of strengthening the apron safety management become more intense.

Many international and domestic scholars have carried out relative researches and have obtained good results. Ciavarelli, A analyses the apron event reason, and presents that more than half of the apron security accidents are caused by the attendants' mistakes[3] . Lee, W.K. develops a quantitative model for assessing aviation safety risk factors as a means of increasing the effectiveness of safety risk management system by integrating the fuzzy linguistic scale method, failure mode, effects and criticality analysis principle, and as low as reasonably practicable approach [7]. But in this article, there has not established the assessment model of airport security risk [7]. Aghahowa and Stephen Allen [1] develops key performance indicators for airport safety and security, and points out that the risk management including risk recognition, risk identification and risk audit, the use of early warning signal and the risk source identification are the important parts of risk management. In 2008, a grey evaluation model for airport apron safety was presented using grey analysis and analytic hierarchy process, the first level indices being the safety of apron facilities, airport public security, management of apron operation

* Corresponding Author 
personnel, management of vehicles in the apron, field management and coordinating ability, and other safety factors [8]. Christina Near, et. al., [5] built aviation safety warning system by using social technology-safety MS model. Sun Dian-ge [13] introduce the Bow-tie risk technology, and present concrete implementation steps of this technology in the civil airfield safety risk. This technology has collected the fault tree, the event tree and the round onion chart theory unifies. Zhao Gui-hong [15] apply the artificial neural network to the apron safety management, they use factor analysis to extract the common factors, and use artificial neural network to train and make prediction of apron safety, finally get the safety condition of apron. Ye Wei [14] established the apron security risk multistage fuzzy comprehensive evaluation model according to the theory of fuzzy mathematics, and had carried on the instance of model validation. Zhang Xiao-quan [16] analyses accident data for a domestic international airport apron in recent five years to identify several types of its most common accidents. And then the Accidents Tree Analysis was applied to analyze the reasons, which were summarized as the number of risk factors, and using grey correlation degree analysis in this sort of important points, such as the working person, the environment, the equipment and the management in the proposed control measures, and finally some feasible suggestions were put forward. Sabine [12] proposes a model, which provides a framework for integrated risk assessments of airport operations. Finally, a macroscopic scenario tool that supports the management of change, training and education, and safety communication functions of the SMS is introduced. LUO Fan [9] sets up a warning simulation model of controlling risk by using flow diagram, based on the comparative analysis of the advantages of system dynamics in the application of pre-warning decision. Pan Wei-jun [10] apply Grey Correlation Theory to make a correlation degree analysis on a civil airport apron's unsafe accidents, according to the structural hierarchical model, obtain the general importance of each factor. Jacek [6] proposed method which reflects the problems of decision making when both objective (represented using non-fuzzy expressions) and subjective (represented using linguistic expressions) criteria, are involved. It has been used to illustrate the problem of attempting to rank in significance the causes of the serious aircraft incident that actually occurred at the F Chopin Airport in Warsaw.

According to the research articles above, up to now, there are many researches on the evaluation of airport risk, but the research on the apron safety risk assessment is less, so a new method of THEIL index and the gray relative analysis is proposed to evaluate precise the apron safety risk. It provides a new way for the apron risk evaluation management.

\section{Materials and Methods}

\subsection{Apron Safety Risk Evaluation Index System}

The skeleton of the evaluation model is the evaluation index system, so establishing a scientific evaluation index system is the key of the successful evaluation work. If the unsuitable evaluation indicators are chosen, or key indicators are lack, the accuracy of evaluation conclusion will be affected; even the error guidance signal is given. In order to be a comprehensive evaluation of apron safety risk, based on the analysis of the related references, a specific apron safety risk evaluation index system is built as shown in Table 1. 
Table 1. Apron Safety Risk Evaluation Index System

\begin{tabular}{c|c}
\hline first-level index & second-level index \\
\hline Environmental factors & $\begin{array}{c}\text { Nature Environmental factors C1 } \\
\text { Artificial environment factors C2 }\end{array}$ \\
Management factors & $\begin{array}{c}\text { The rationality of the control process C3 } \\
\text { The perfect degree of the rules and regulations C4 } \\
\text { The apron operation vehicle usage and management } \\
\text { standardization C5 }\end{array}$ \\
\hline Equipment and facilities & $\begin{array}{c}\text { Scheduling of equipment and facilitiesC6 } \\
\text { The reliability of the apron facilities and maintenance C7 }\end{array}$ \\
\hline Human factor & $\begin{array}{c}\text { Staff health C8 } \\
\text { Personnel technical level C9 } \\
\text { Work attitude C10 }\end{array}$ \\
\hline
\end{tabular}

\subsection{Process of THEIL Index and the Gray Connection Analysis Trust Evaluation for Sensor}

In the evaluation of airport apron safety risk, the main idea of the model is: according to the basic thought of THEIL imbalance index, using the THEIL index to calculate the unbalanced degree of each evaluation index. The unbalanced degree of evaluation index reflects dispersion between different airport apron safety risk evaluation index values, which presents the influence of the index to the comprehensive evaluation result. Then the objective weights of the indexes are calculated by using the unbalanced degree of the evaluation index, combined with subjective weights by the expert experience, the comprehensive index weights are obtained. Based on above comprehensive weights, the fuzzy evaluation and grey correlation are used to obtain the results of degree of association, and specific risk evaluation order of aprons is achieved.

Evaluation model of data preprocessing: For multiple attribute decision-making problems, assuming that $X=\left\{x_{1}, x_{2}, \cdots, x_{n}\right\}$ as an object or set of solutions, $U=\left\{\boldsymbol{u}_{1}, \boldsymbol{u}_{2}, \cdots, \boldsymbol{u}_{m}\right\}$ as attribute set, $\boldsymbol{w}=\left(\boldsymbol{w}_{1}, \boldsymbol{w}_{2}, \cdots, \boldsymbol{w}_{m}\right)^{T}$ as the weight vector of attributes, among them, $w_{i} \geq 0, \sum_{i=1}^{m} w_{i}=1$. For an object or scheme $x_{j} \in X$, measure according to article ${ }^{i}$ attribute ${ }^{u_{i}}$, get $x_{j}$ on the value of the $u_{i}$ attribute, according to the type of attribute ${ }^{u_{i}}$, a property can be formed in a qualitative or quantitative, on different parameters values are normalized according to the extreme value processing method, normalized index denoted as $a_{i j}$, then the model has the characteristic matrix: $A=\left(a_{i j}\right)_{m \times n}$.

Comprehensive weighting: The first step, on the standardization of the decision matrix $A=\left(a_{i j}\right)_{m \times n}$, according to the unbalanced THEIL index, for the evaluation index $i$, the unbalanced degree $T_{i}$ is:

$$
T_{i}=\ln n+\sum_{j=1}^{n}\left(\frac{a_{i j}}{\sum_{j=1}^{n} a_{i j}} \ln \frac{a_{i j}}{\sum_{j=1}^{n} a_{i j}}\right), i=1,2, \ldots, m
$$


The second step, on the basis of the above unbalanced index $T_{i}$, the index weight of indicators is defined:

$$
p_{i}=\frac{T_{i}}{\sum_{i=1}^{m} T_{i}}, i=1,2, \ldots, m
$$

The third step, D according to the decision maker's experience knowledge, the subjective weights of each attribute in the conditional attribute set are given, namely $Q_{i}(i=1,2, . ., m)$. Among them,

$$
\sum_{i=1}^{n} Q_{i}=1
$$

The forth step, the appropriate experience factor $\alpha$ is chosen, and then according to the formula, the comprehensive weight of each index attribute ${ }^{x_{i}}$ is calculated:

$$
w_{i}=\alpha Q_{i}+(1-\alpha) p_{i}(1 \leq i \leq m)
$$

Fuzzy comprehensive evaluation method: The fuzzy comprehensive evaluation method is a kind of systems analysis method which uses the principle of fuzzy mathematics to analyze and evaluate "fuzzy" things. It is a qualitative and quantitative fuzzy inference-based combination of precision and non-precision unity of analysis and evaluation method. Due to the unique advantages of this method demonstrated in dealing with complex systems problems that cannot be described by precise mathematical method, it has been widely applied to many subject areas in recent years.

Given two limited theory field:

$$
\begin{gathered}
U=\left\{U_{1}, \ldots, U_{m}\right\} \\
V=\left\{v_{1}, \cdots, v_{n}\right\}
\end{gathered}
$$

Among them, $\mathrm{U}$ represents all the evaluation factors sets; $V$ represents all the comments level sets. If you focus on the evaluation factor $u_{i}, i(i=1,2, \ldots, m)$, the single factor evaluation results is $R=\left\{r_{i 1}, r_{i 2}, \cdots, r_{i n}\right\}$. If the numbers of evaluation factor are $m$, the evaluation decisions matrix of these evaluation factors is:

$$
\mathrm{R}=\left(\begin{array}{c}
R 1 \\
R 2 \\
\cdot \\
R m
\end{array}\right)=\left(\begin{array}{cccc}
r_{11} & r_{12} & \ldots & r_{1 n} \\
r_{21} & r_{22} & \ldots & r_{2 n} \\
\cdot & \cdot & . & \cdot \\
r_{m 1} & r_{m 2} & \ldots & r_{m n}
\end{array}\right)
$$

A fuzzy relation is $\mathrm{U}$ to $\mathrm{V}$.

If the Weight distribution for each evaluation factors is $\stackrel{A}{\approx}=\left[a_{1}, a_{2}, \cdots, a_{m}\right]$ (Apparently $\stackrel{A}{\sim}$ is a fuzzy subset in domain of discourse $U$, and $0 \leqslant a_{i} \leqslant 1, \sum_{i=1}^{m} a_{i}=1$, then, applying of fuzzy transformation synthesis operation, we can get a fuzzy subset in domain of discourse $\mathrm{V}$, namely, the comprehensive evaluation results: $B=A o R=\left[b_{1}, b_{2}, \ldots, b_{n}\right]$.

After the synthesis weights of indicators are calculated by using THEIL index, a weighted average of the model of the fuzzy comprehensive evaluation method is used to 
form comprehensive evaluation Matrix $B$. But the results did not give a specific order, so it is necessary to adopt the method of grey correlation correction based on evaluation results, this determines the final sorting of the results.

Grey correlation analysis: Grey correlation analysis is one of the main content of the grey system analysis. This method can determine the degree of correlation between factors based on various factors change curve geometric shape similarity degree. The basic idea of it is: apply dimensionless method to the original observation evaluation indicators, calculate correlation coefficient, correlation and rank the evaluation indexes according to the size of the correlation.

Steps of grey relation analysis are as follows:

The first step is to determine the analysis sequence.

Set the reference sequence (also known as the mother sequence) is $Y=\{Y(k) \mid k=1,2, \cdots, n\}, \quad$ comparison sequence is $\quad X_{\mathrm{i}}=\left\{X_{i}(k) \mid k=1,2, \cdots, n\right\}$, $i=1,2, \cdots, m$.

The second step, carry on the dimensionless process.

Due to various factors in the system data have the different dimensions; it is difficult to get the correct conclusion when they are compared. So the grey correlation analysis generally must carry on the dimensionless processing of data.

$$
x_{i}(k)=\frac{X_{i}(k)}{X_{i}(1)}, k=1,2, \cdots, n ; i=0,1,2, \cdots, m
$$

The third step, calculate correlation coefficient.

Correlation coefficient of $x_{0}(k)$ and $x_{i}(k)$

$$
\xi_{i}(k)=\frac{\min _{i} \min _{k}\left|y(k)-x_{i}(k)\right|+\rho \max _{i} \max _{k}\left|y(k)-x_{i}(k)\right|}{\left|y(k)-x_{i}(k)\right|+\rho \max _{i} \max _{k}\left|y(k)-x_{i}(k)\right|}
$$

Namely $\Delta_{i}(k)=\left|y(k)-x_{i}(k)\right|$.So

$$
\xi_{i}(k)=\frac{\min _{i} \min _{k} \Delta_{i}(k)+\rho \max _{i} \max _{k} \Delta_{i}(k)}{\Delta_{i}(k)+\rho \max _{i} \max _{k} \Delta_{i}(k)}
$$

$\rho \in(0, \infty)$, Called identification coefficient. The smaller $\rho$ is, the greater the resolution is. General, $\rho$ value interval $(0,1)$, the specific values can be seen as local conditions. When $\rho \leq 0.5463$, the resolution is best, so we can choose $\rho=0.5$.

The fourth step, calculate grey correlation degree.

Correlation degree of reference sequence and comparison sequence:

$$
r_{i}=\frac{1}{n} \sum_{k=1}^{n} \xi_{i}(k) \quad, k=1,2, \cdots, n .
$$

Fifth, rank the correlation degree.

According to the value of grey correlation degree, each object will be sorted. If $r_{1}<r_{2}$, reference sequence ${ }^{y}$ is more similar to comparison sequence ${ }^{x_{2}}$. In other words, the value is larger, the assessment is better. 


\section{Results and Discussion}

Experiment results and analysis: Based on the risk evaluation index system and evaluation model above, we select four airport aprons in central China as the research objects to analyze.

\subsection{Calculation Process}

First of all, according to indicators c1-c10, adopted the data processing method of the normalization, normalization of index are shown in Table 2.

Table 2. Evaluation Index Value

\begin{tabular}{lllllllllll}
\hline & C1 & C2 & C3 & C4 & C5 & C6 & C7 & C8 & C9 & C10 \\
\hline Apron A1 & 0.56 & 0.55 & 0.76 & 1 & 0.74 & 0.78 & 0.51 & 0.88 & 0.61 & 0.75 \\
Apron A2 & 0.68 & 1 & 0.45 & 0.76 & 0.86 & 0.78 & 0.84 & 0.8 & 0.61 & 1 \\
Apron A3 & 1 & 0.89 & 0.87 & 0.88 & 0.56 & 1 & 1 & 0.87 & 1 & 0.74 \\
\hline Apron A4 & 1 & 0.77 & 1 & 0.44 & 0.78 & 1 & 0.76 & 0.55 & 0.67 & 1 \\
\hline
\end{tabular}

According to formula (1)-(2), calculate the weights of indicators

$p_{i}=(0.1307,0.0999,0.1665,0.1762,0.0514,0.0339,0.1198,0.0702,0.1041$, 0.0474). Suppose the decision-maker's subjective evaluation criterion weight $Q_{i}(i=1,2, . ., n)=(0.1,0.08,0.2,0.12,0.1,0.05,0.1,0.1,0.1,0.05)$, experience factor is 0.7 , According to formula (3), calculate the normalization comprehensive weighting:

$$
\begin{gathered}
w_{j}=(0.1092,0.0860, \quad 0.1899,0.1369,0.0854,0.0452,0.1059,0.0911,0.1012, \\
0.0492)
\end{gathered}
$$

Next, initial apron security risk assessment data are analyzed, the decision results are obtained. There are 10 experts to evaluate the four airport apron safety risk; the initial evaluation data are processed. Each index was divided into 3 grades from low risk to high risk. Quantitative statistics data of the apron A1 are shown in Table 3. In Table 3, the evaluation result of each index is the proportion of 10 experts to choose three grades on this index evaluation. Take index $C_{1}$ as example, expert proportion of Choose "low risk" is $80 \%$, proportion of Choose "general risk" is $20 \%$, proportion of Choose "high risk" is $0 \%$.

Table 3. Statistics Data of Apron A1

\begin{tabular}{c|c|c|c|c}
\hline evaluation index & weights & \multicolumn{3}{|c}{ Apron A1 } \\
\cline { 3 - 5 } & w & Low risk & General risk & High risk \\
\hline$C_{1}$ & 0.1092 & 0.8 & 0.2 & 0 \\
\hline$C_{2}$ & 0.0860 & 0.8 & 0.1 & 0.1 \\
\hline$C_{3}$ & 0.1899 & 0.5 & 0.4 & 0.1 \\
\hline$C_{4}$ & 0.1369 & 0.4 & 0.4 & 0.2 \\
\hline$C_{5}$ & 0.0854 & 0.7 & 0.2 & 0.1 \\
\hline$C_{6}$ & 0.0452 & 0.7 & 0.3 & 0 \\
\hline$C_{7}$ & 0.1059 & 0.5 & 0.3 & 0.2 \\
\hline$C_{8}$ & 0.0911 & 0.7 & 0.2 & 0.1 \\
\hline$C_{9}$ & 0.1012 & 0.8 & 0 & 0.2 \\
\hline$C_{10}$ & 0.0492 & 0.9 & 0.1 & 0 \\
\hline
\end{tabular}




\subsection{Results and Discussion}

To apron A1, a weighted average of the model of the fuzzy comprehensive evaluation method is used to form comprehensive evaluation:

$$
A 1=w_{i} \cdot R=(0.639,0.247,0.114)
$$

The same procedure may be easily adapted to obtain other three airport apron evaluation results:

$$
\begin{aligned}
& A 2=w_{i} \cdot R=(0.578,0.254,0.168) \\
& A 3=w_{i} \cdot R=(0.249,0.502,0.249) \\
& A 4=w_{i} \cdot R=(0.285,0.548,0.167)
\end{aligned}
$$

Conclusion by the maximum membership degree: the apron A1 and A2 evaluation "low risk", the apron A3 and A4 evaluation "general risk". But a clear ultimate classification is not given. For example, apron A3 and A4 evaluation are "general risk", but which apron evaluation is the best has not determined. Now, we use the grey relate degree to improve conclusion. At last, we reached classification. Four airport apron fuzzy evaluation results and the ideal reference data series are shown in Table 4, after the calculation steps of grey association analysis, the correlation coefficient and correlation and rank are obtained as shown in Table 5 .

Table 4. Four Airport Apron Fuzzy Evaluation Results and the Ideal Reference Data Series

\begin{tabular}{c|c|c|c}
\hline Apron & Low risk & General risk & High risk \\
\hline Apron A1 & 0.639 & 0.247 & 0.114 \\
\hline Apron A2 & 0.578 & 0.254 & 0.168 \\
\hline Apron A3 & 0.249 & 0.502 & 0.249 \\
\hline Apron A4 & 0.285 & 0.548 & 0.167 \\
\hline Ideal reference sequence & 1 & 0.548 & 0 \\
\hline
\end{tabular}

Table 5. The Correlation Coefficient and Correlation and Rank of 4 Apron Risk Evaluation

\begin{tabular}{c|c|c|c|c|c}
\hline Apron & \multicolumn{3}{|c|}{ correlation coefficient } & Correlation degree & rank \\
\hline Apron A1 & 1 & 0.8371 & 0.5220 & 0.7864 & 1 \\
\hline Apron A2 & 0.8397 & 0.8554 & 0.3333 & 0.6762 & 2 \\
\hline Apron A3 & 0.4503 & 0.5760 & 0.3333 & 0.4532 & 4 \\
\hline Apron A4 & 0.4744 & 0.5252 & 0.4271 & 0.4756 & 3 \\
\hline
\end{tabular}

The Table 5 shows that, among four airport apron safety risk assessment results, the apron $\mathrm{A} 1$ is minimum risk, the apron $\mathrm{A} 3$ is the highest risk.

Through unbalanced degree of the evaluation index (namely the index itself information) to determine the weights of evaluation indexes, then based on this weight, fuzzy evaluation model is built. Finally, grey evaluation is used to modify this model. When system associated factors are analyzed, compared with some mathematical statistics methods such as regression analysis, variance analysis, principal component analysis and so on, the grey relation analysis method has unique advantages. It is equally applicable for the sample more or less and the sample irregular. This method make the evaluation results more objective and reasonable, to a certain extent, it improve and enhance the quality of the comprehensive evaluation. 


\section{Conclusion}

Precise sort problem of the airport apron risk evaluation is solved by using THEIL index -grey association analysis decision model in this paper. According to the basic thought of THEIL imbalance index, using the THEIL index to calculate the unbalanced degree of each evaluation index. Then the objective weights of the indexes are calculated by using the unbalanced degree of the evaluation index, combined with subjective weight by the expert experience, the comprehensive index weight is obtained. Based on above comprehensive weight, the fuzzy evaluation and grey correlation are used to obtain the results of degree of association, and specific risk evaluation order of aprons is achieved. In practice, we can setup a computer-program which can enhance work efficiency and accuracy greatly for evaluation.

\section{Acknowledgements}

This study is supported by the National Nature Science Foundation (71271163) in China. The author would like to thank for it, under which the present work was possible.

\section{References}

[1] A. Enoma and S. Allen, "Developing key performance indicators for airport safety and security", Journal of Air Transport Management, vol. 25, no. 7, (2007), pp. 296-314.

[2] B. Kaiser, C. Gramlich and M. Forester, "State/Event Fault Trees- a Safety Analysis Model for Software- controlled System", Reliability Engineering and System Safety, vol. 92, no. 2, (2007), pp. 1521-1537.

[3] A. Ciavarelli Jr. and R. Figlock, "Organizational factors in aviation accidents", International Journal of Aviation Psychology, vol. 16, no. 3, (2006), pp. 239-261.

[4] C. L. de la Vegaa and O. Volijb, "A simple proof of Foster's (1983) characterization of the Theil measure of inequality", Economic Modeling, vol. 35, (2013), pp. 940-943.

[5] C. Neal, O. Girina, S. Senyukov, A. Rybin and J. Osiensky, "Russian eruption warning systems for aviation", Natural Hazards, vol. 51, no. 2, (2009), pp. 245-262.

[6] J. Skorupski, "Multi-criteria group decision making under uncertainty with application to air traffic safety", Expert Systems with Applications, vol. 41, (2014), pp. 7406-7414.

[7] Lee W. K., "Risk assessment modeling in aviation safety management", Journal of Air Transport Management, vol. 12, no. 5, (2006), pp. 267-273.

[8] Liu G., Zhu j.f.., "Grey analysis of airport apron safety", Journal of Southwest Jiaotong University, vol. 43, no. 5, (2008), pp. 600-604.

[9] Luo F., Liu z.p. and Yang Z, "Pre-warning Decision-making Model of Air Traffic Control Safety Risk based on System", System engineering, vol. 32, no. 1, (2014), pp. 139-144.

[10] Pan w.j. and Qiu w.b., "Airport Apron Safety Management Research", Science Technology and Industry, vol. 14, no. 1, (2014), pp. 133-135,170.

[11] Qian x.s., Yu j.y. and Dai r.w., "A new discipline of science - The study of open complex giant system and its methodology", The journal nature, vol. 13, no. 1, (1990), pp. 3-10.

[12] S. Wilke, A. Majumdar and W. Y. Ochieng, "Airport surface operations: A holistic framework for operations modeling and risk management", Safety Science, vol. 63, (2014), pp. 18-33.

[13] Sun d.g., Sun J., Wang M. and Qin K., 2010, "Application of the improved Bow-tie risk analysis technology in civil airport safety", Journal of Safety Science and Technology, vol. 6, no. 4, (2010), pp. 85-90.

[14] Y. Wei, Huang t.b.. "Renovated multilevel fuzzy in the grated hazards assessment system for the airport apron safety", Journal of Safety and Environment, vol. 11, no. 3, (2011), pp. 231-235.

[15] Zhao g.h.. "Application of artificial neural network in apron's pro-warning", Journal of Civil Aviation University of China, vol. 6, vol. 29, (2011), pp. 31-34.

[16] Zhang x.q. and Liu x., "Study on risk management of airport apron", Journal of Safety Science and Technology, vol. 7, no. 3, (2011), pp. 42-47. 

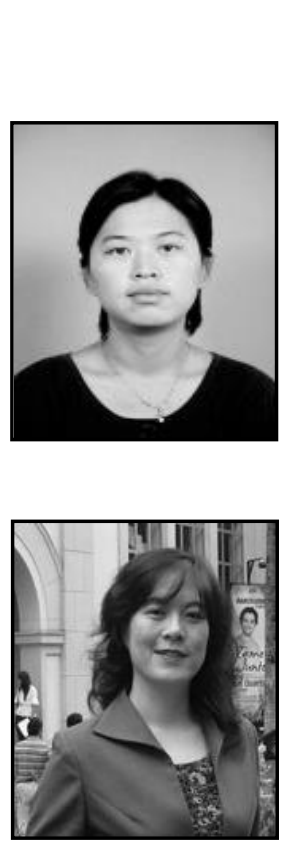

\section{Authors}

Li Shi, Born in Hubei in 1980, she is an associate professor of Hubei University of economics; a postdoctoral researcher of Wuhan University of Technology; a visiting scholar of Department of Management Science and Information Systems, Rutgers University, New Jersey. She has authored a number of different journal and paper. Her research interests include service science, data mining, and decision support. She is a member of CCF.

Fan Luo, Born in Yiyang, Hunan in 1963, she is now a professor, supervisor of doctoral student and deputy director of the department of Human Resource Management in Wuhan University of Technology. Her main research direction includes Human resources development and management, risk early warning and emergency management. 
International Journal of Security and Its Applications

Vol. 10, No. 7 (2016) 